Ann. Zootech., I972, 21 (2), 207-222.

\title{
MATIÊRES GRASSES VÉGÉTALES ET ANIMALES DANS LES ALIMENTS D'ALLAITEMENT POUR VEAUX A L'ENGRAIS
}

\author{
B.-G, CotTyn, M.-R. CASTeEls, P.-R. MESkens * et F.-X. BUySSE \\ avec la collaboration technique de G. Vanooteghem et J. VAN Hecke \\ Station de Recherches pour l'Alimentation du Bétail, Gontrode, Belgique. \\ Divecteur : $F .-X$. Buysse \\ * Laboratoires Palmafina-Bruxelles
}

\section{RÉSUMÉ}

Sur 5 groupes de 8 veaux à l'engrais de races Pie-rouge de Flandre Orientale et Pie-noire les auteurs ont étudié l'influence de différentes matières grasses incorporées à raison de 20 p. Ioo dans des aliments d'allaitement, sur la croissance, l'efficacité alimentaire et la composition de la carcasse.

Dans les deux premiers aliments, les matières grasses employées étaient exclusivement du suif ou du saindoux. Dans les trois autres elles étaient constituées par des mélanges contenant respectivement : Io $\mathrm{p}$. Ioo d'huile de baleine hydrogénée, I 5 p. roo d'huile de palme, $25 \mathrm{p}$. Ioo d'huile de coprah et 50 p. Ioo de suif (BPCS) - I 5 p. Ioo d'huile de palme, $25 \mathrm{p}$. Ioo d'huile de coprah et $60 \mathrm{p}$. Ioo d'huile de baleine hydrogénée (PCB) — Io p. Ioo d'huile de soja, i 5 p. roo d'huile de palme, i 5 p. Ioo de suif et 60 p. Ioo d'huile de baleine hydrogénée (SoPSB).

Les veaux ont été abattus après une période expérimentale moyenne de 124 jours et les poids moyens au début et à fin ont été respectivement de 43 et $\mathrm{r} 80 \mathrm{~kg}$.

La meilleure croissance moyenne journalière ( 1 I69 g) et l'indice de consommation le plus favorable $(\mathrm{I}, 56 \mathrm{~kg}$ d'aliment $/ \mathrm{kg}$ de gain de poids) ont été obtenus avec le mélange PCB. Avec le mélange BPCS la croissance journalière a été un peu moindre ( $\begin{array}{lll}1 & 55 & \mathrm{~g}\end{array}$ ) et l'indice de consommation quasi identique $(\mathrm{I}, 57)$. La suppression de l'huile de coprah dans le mélange et son remplacement partiel par de l'huile de soja (SoPSB) a diminué légèrement la croissance journalière ( 1 I $16 \mathrm{~g}$ ) tout en influençant défavorablement l'indice de consommation ( 1,64$)$. La croissance journalière la moins élevée a été obtenue avec l'aliment renfermant le saindoux pur ( $\mathrm{I}$ o24 $\mathrm{g}$ au lieu de $\mathrm{I}$ o66 g avec l'aliment contenant le suif). La croissance journalière obtenue avec le saindoux a été significativement inférieure à celles réalisées avec les mélanges PCB et BPCS. Avec le suif et le saindoux, l'indice de consommation a été significativement moins satisfaisant qu'avec les mélanges PCB et BPCS. Grâce à l'indice de consommation sensiblement plus favorable obtenu avec ces deux derniers mélanges, les couts alimentaires par kg de gain de poids ont été, avec ceux-ci nettement plus bas (respectivement 27,6 et 28, I F. B.) qu'avec le suif, le saindoux et le SoPSB (uniformément 29, Io F. B.). Il n'y a pas eu de différences significatives entre les 5 traitements en ce qui concerne le rendement à l'abattage et la qualité de la viande. Les veaux qui recevaient les aliments contenant le suif ou le saindoux ont eu des graisses de dépôt moins fermes que ceux qui consommaient les aliments contenant les mélanges de matières grasses. Les acides gras à chaîne moyenne (de $\mathrm{C}_{8}$ à $\mathrm{C}_{12}$ inclus) de même que les acides gras polyinsaturés, ont été peu stockés dans les graisses de dépôt, contrairement aux acides gras longs saturés. 


\section{I. 一 INTRODUCTION}

A cause de son alimentation exclusivement à base de produits laitiers, le veau à l'engrais se comporte comme un monogastrique fonctionnel. Du point de vue technique et à cause de sa composition équilibrée en éléments nutritifs, le lait entier constitue l'aliment idéal pour ce type de production de viande. Pour des motifs économiques, le lait entier a cependant été remplacé en grande partie par les alimants d'allaitement. Ces aliments, tels qu'ils se sont développés au cours des années, sont composés de 60 à $70 \mathrm{p}$. Ioo de produits laitiers (principalement de poudre de lait écrémé), auxquels viennent s'ajouter des matières grasses, du dextrose. de l'amidon, des minéraux, des vitamines et des additifs. Pour des raisons économiques, les matières grasses les plus couramment employées sont surtout le suif et le saindoux. Du point de vue physiologique, 1'utilisation d'autres matières grasses végétales ou animales, telles que l'huile de baleine partiellement hydrogénée, l'huile de coprah, l'huile de palme et l'huile de soja, peut également être envisagée. La composition en acides gras (longueur de chaîne, degré d'insaturation et position dans la molécule triglycéridique) a une importance prépondérante sur l'utilisation digestive des matières grasses par le veau (Touldec et Mathieu, ig69).

Dans 1'essai rapporté ici, nous avons comparé 5 aliments d'allaitement qui ne différaient entre eux que par la composition des matières grasses. En plus de 2 aliments ne renfermant que du suif ou du saindoux, 3 autres ont été préparés avec des combinaisons d'huile de baleine partiellement hydrogénée, d'huile de coprah, d'huile de palme, d'huile de soja et de suif. Outre les aspects pratiques de production de viande, nous avons également étudié les critères de qualité des matières grasses employées, ainsi que l'influence de ces dernières sur la composition des graisses de dépôt. Etant donné que plus que la conformation et l'état d'engraissement, la cou1leur de la viande étant le principal facteur dans la détermination du prix payé pour les veaux gras, l'incidence de ces différentes formules sur la couleur de la viande a été également déterminée.

\section{II. - MATÉRIEL ET MÉTHODES}

\section{Animaux}

Pour cet essai, nous avons utilisé to veaux mâles de race Pie-rouge de la Flandre Orientale et de race Pie-noive, achetés dans le commerce à l'âge d'environ 7 jours. Ces animaux ont été logés dans des cases individuelles sur caillebotis non paillés ( $140 \times 60 \mathrm{~cm}$ ) dans une étable ventilée et chauffée en période hivernale. Avant le début de l'essai, les veaux ont été divisés en cinq groupes comparables, compte tenu de leur poids, de la valeur de leur hématocrite et de la teneur en hémoglobine de leur sang, ces deux derniers critères étant déterminants pour la couleur finale de la viande (Charpentier, ig66; Verbeke et Martin, i967 ; Eeckhout et Casteels, i969).

\section{Régime alimentaire}

Au moyen d'un mélangeur rapiđe, les aliments d'allaitement ont été dilués dans đe l'eau à $45^{\circ} \mathrm{C}$ au moment des repas ( 2 par jour).

Les laits de remplacement ainsi obtenus ont été distribués aux veaux, dans des seaux indi- 
viduels, à une température d'environ $37^{\circ} \mathrm{C}$. La quantité journalière d'aliment d'allaitement, qui était de $300 \mathrm{~g}$ au début de l'essai augmentait graduellement suivant un plan déterminé pour atteindre 3 ooo g par jour à la fin de la période d'engraissement. La concentration du lait de remplacement, qui était de $\mathrm{I} 5 \mathrm{O} \mathrm{g}$ de poudre par litre d'eau les I I premières semaines, était portée à $\mathrm{I} 75 \mathrm{~g}$ à partir de la $\mathrm{I}^{\mathrm{e}}$ semaine. Les veaux étaient pesés toutes les 4 semaines. L'essai a duré, en moyenne environ, $\mathbf{1} 7,5$ semaines et le poids moyen des veaux était alors de $\mathbf{1} 80 \mathrm{~kg}$.

\section{Composition des aliments}

La composition des aliments d'allaitement est rapportée dans les tableaux $\mathbf{I}$ et 2 . Les matières grasses ont été incorporées à raison de $20 \mathrm{p}$. Ioo ; dans 2 des aliments, elles étaient apportées exclusivement par du suif ou du saindoux purs, tandis que dans les 3 autres, elles étaient constituées par des mélanges contenant une proportion constante (I 5 p. Ioo) d'huile de palme raffinée et Io p. roo (BPCS) ou 6 o p. Ioo (PCB et SoPSB) d'huile de baleine hydrogénée. Ces 3 mélanges renfermaient, en outre, soit $25 \mathrm{p}$. Ioo d'huile de coprah raffinée (BPCS et PCB), soit une combinaison d'huile de soja raffinée (ro p. IOO) et de suif (15 p. IOO); le mélange BPCS renfermait par ailleurs $5 \circ \mathrm{p}$. roo de suif.

TABLEAU I

Composition des aliments d'allaitement (p. 100)

\begin{tabular}{|c|c|c|c|c|c|}
\hline Groupe expérimental & Suif & Saindoux & BPCS & PCB & SoPSB \\
\hline $\begin{array}{l}\text { Poudire de lait écrémé (spray) } \\
\text { Poudre de lactosérum } \ldots \ldots \ldots \\
\text { Amidon } \ldots \ldots \ldots \ldots \ldots \ldots \\
\text { Glucose (dextrose) } \ldots \ldots \ldots \ldots\end{array}$ & $\begin{array}{r}63 \\
7 \\
5 \\
3\end{array}$ & $\begin{array}{r}63 \\
7 \\
5 \\
3\end{array}$ & $\begin{array}{r}63 \\
7 \\
5 \\
3\end{array}$ & $\begin{array}{r}63 \\
7 \\
5 \\
3\end{array}$ & $\begin{array}{r}63 \\
7 \\
5 \\
3\end{array}$ \\
\hline 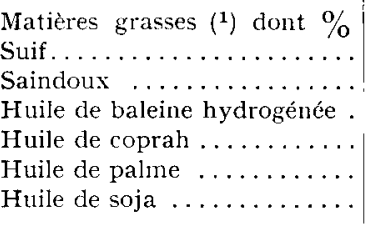 & $\begin{array}{c}20 \\
(100)\end{array}$ & $\begin{array}{r}20 \\
(100)\end{array}$ & $\begin{array}{l}20 \\
(50) \\
(10) \\
(25) \\
(15)\end{array}$ & $\begin{array}{l}20 \\
(60) \\
(25) \\
(15)\end{array}$ & $\begin{array}{l}20 \\
(15) \\
(60) \\
(15) \\
(10)\end{array}$ \\
\hline $\begin{array}{l}\text { Saccharoglycérides }\left(^{2}\right) \quad \ldots \ldots \\
\text { Lécithine de soja } 65 \text { p. } 100 \ldots \\
\text { Complément vitaminisé et } \\
\text { minéral }\left(^{3}\right) \ldots \ldots \ldots \ldots \ldots \ldots \\
\text { Terramycine } \ldots \ldots \ldots \ldots \ldots \ldots\end{array}$ & $\begin{array}{l}0,620 \\
0,280 \\
1 \\
0,005\end{array}$ & $\begin{array}{l}0,620 \\
0,280 \\
1 \\
0,005\end{array}$ & $\begin{array}{l}0,620 \\
0,280 \\
1 \\
0,005\end{array}$ & $\begin{array}{l}0,620 \\
0,280 \\
1 \\
0,005\end{array}$ & $\begin{array}{l}0,620 \\
0,280 \\
1 \\
0,005\end{array}$ \\
\hline
\end{tabular}

(1) Antioxydants : BHT (2/10 000)

(2) Émulsifiant : par $100 \mathrm{~g}$ de matières grasses : 3,1 g de MST et 1,4 $\mathrm{g}$ de lécithine de soja

(3) Par $100 \mathrm{~kg}$ d'aliment d'allaitement

Vitamine $\begin{array}{ll}\mathrm{A}: 3800000 \mathrm{UI} & \text { Pantothénate de Ca:630 mg } \\ \mathrm{D}_{3}: 380000 \mathrm{UI} & \text { Acide nicotinique:4,75 g } \\ \mathrm{C}: 9 \mathrm{~g} & \text { Chlorure de choline }: 28,5 \mathrm{~g} \\ \mathrm{~B}_{1}: 210 \mathrm{mg} & \mathrm{ZnO}: 5,2 \mathrm{~g} \\ \mathrm{~B}_{2}: 570 \mathrm{mg} & \mathrm{CuO}: 2,34 \mathrm{~g} \\ \mathrm{~B}_{6}: 95 \mathrm{mg} & \mathrm{MgO}: 81 \mathrm{~g} \\ \mathrm{~B}_{12}: 1,4 \mathrm{mg} & \mathrm{NaCl}: 150 \mathrm{~g} \\ \mathrm{E}: 1,9 \mathrm{~g} & \mathrm{CaHPO} \cdot 2 \mathrm{H}_{2} \mathrm{O}: 300 \mathrm{~g}\end{array}$




\section{TABLEAU 2}

Analyse chimique (p. 1oo) des 5 rations

\begin{tabular}{|c|c|c|c|c|c|}
\hline Groupe expérimental & Suif & Saindoux & BPCS & PCB & SoPSB \\
\hline Matière sèche $\ldots \ldots \ldots$ & 96,9 & 96,6 & 96,4 & 96,6 & 95,8 \\
\hline Matières azotées...... & 23,3 & 23,0 & 23,0 & 23,3 & 23,0 \\
\hline Matières grasses ...... & 20,9 & 20,6 & 20,7 & 20,4 & 20,4 \\
\hline Cendres $\ldots \ldots \ldots \ldots$. & 6,3 & 6,3 & 6,3 & 6,3 & 6,2 \\
\hline Extractif non azoté. & $46, t_{4}$ & 46,7 & 46,4 & 46,6 & $l_{1} 6,2$ \\
\hline $\mathrm{Fe}$ (p.p.m.) $\ldots \ldots$ & 15 & 17 & 14 & 19 & 13 \\
\hline
\end{tabular}

\section{TABLEAU 3}

Qualité des matières grasses alimentaires (I) et des graisses de dépót correspondantes (2) (*)

\begin{tabular}{|c|c|c|c|c|c|c|c|c|c|c|}
\hline \multirow{2}{*}{$\begin{array}{c}\text { Composition } \\
\text { des matières grasses }\end{array}$} & \multicolumn{2}{|c|}{ Suif } & \multicolumn{2}{|c|}{ Saindoux } & \multicolumn{2}{|c|}{ BPCS } & \multicolumn{2}{|c|}{$\mathrm{PCB}$} & \multicolumn{2}{|c|}{ SoPSB } \\
\hline & (1) & (2) & (1) & $(2)$ & (1) & $(2)$ & (1) & $(\underline{a})$ & (1) & $(2)$ \\
\hline Acidité oléique $\ldots \ldots$ & 0,048 & 1,55 & 0,05 ; & 2,10 & 0,056 & 1,69 & 0,099 & 1,40 & 0,100 & 1,79 \\
\hline Indice d'iode $\ldots \ldots$ & 46,1 & 52,5 & 57,1 & 57,4 & 41,3 & 49,5 & 54,6 & 49,9 & 62,1 & 56,0 \\
\hline $\begin{array}{l}\text { Indice de saponifi- } \\
\text { cation } \ldots \ldots \ldots \\
\text { Indice de peroxyde }\end{array}$ & 94 & 192 & 195 & 191 & 206 & 195 & 198 & 195 & 188 & 182 \\
\hline$(\mathrm{méq} / \mathrm{kg}) \ldots \ldots$ & 0,7 & 1,7 & 1,0 & 2,3 & 0,6 & 1,1 & 0,6 & 0,8 & 1,0 & 1,9 \\
\hline $\begin{array}{l}\text { Test de stabilité } \\
\text { selon Swift }(+2 / \\
10000 \mathrm{BHT}):\end{array}$ & & & & & & & & & & , \\
\hline $15 \mathrm{~h}, \ldots \ldots$ & 7,5 & - & 6,8 & - & 6,9 & 一 & 6,1 & $\cdots$ & 7,0 & - \\
\hline après $18 \mathrm{~h}, \ldots \ldots$ & 12,7 & 18,6 & 8,1 & 274,0 & 11,5 & 12,9 & 10,1 & 13,9 & 13,2 & 518,2 \\
\hline apres $_{21} \mathrm{~h} \mid \ldots \ldots$ & 10,2 & 30,3 & 9,6 & 412,2 & 8,7 & 40,5 & 16,0 & 23,6 & 14,6 & 644,8 \\
\hline $24 \mathrm{~h}(\ldots \ldots$ & 8,0 & 59,8 & 10,9 & 629,5 & 9,2 & 248,7 & 9,5 & $41, \mathbf{t}$ & 14,1 & 801,6 \\
\hline $\begin{array}{l}\text { Point de fusion (ca- } \\
\text { pillaire ouvert) }\end{array}$ & 43,9 & 38,0 & 37,6 & 35,2 & 37,2 & 37,2 & 37,0 & 37,6 & 37,0 & 35,8 \\
\hline Titre de Dalican ... & 43,0 & 41,1 & 40,7 & 40,2 & 37,7 & 40,7 & 34,4 & 38,7 & 36,2 & 39,7 \\
\hline $\begin{array}{l}\text { MNR (Résonance } \\
\text { magnétique nu- } \\
\text { cléaire) }\left(*^{*}\right):\end{array}$ & & & & & & & & & & \\
\hline $10^{\circ} \mathrm{C} \ldots \ldots$ & 46,41 & 32,87 & 52,26 & 26,13 & 52,52 & 47,17 & 57,28 & 59,83 & 55,88 & 42,92 \\
\hline $20^{\circ} \mathrm{C} \ldots$ & 42,67 & 22,25 & 33,08 & 15,40 & 28,84 & 29,04 & $3\{, 19$ & 43,19 & 36,99 & 27,66 \\
\hline $25^{\circ} \mathrm{C} \ldots \ldots \ldots$ & 29,98 & 11,53 & 20,32 & 5,00 & 18,59 & 16,66 & 20,86 & 28,72 & 23,46 & 17,33 \\
\hline $30^{\circ} \mathrm{C} \ldots \ldots \ldots$ & 20,64 & 3,02 & 5,62 & 0,21 & 9,94 & 8,30 & 8,23 & 15,15 & 11,66 & 6,24 \\
\hline $40^{\circ} \mathrm{C} \ldots \ldots \ldots$ & 6,68 & 0,00 & - & $\longrightarrow$ & - & - & - & - & - & - \\
\hline
\end{tabular}

(*) Absence d'appréciables quantités d'humidité, d'impuretés ou de savons dans les différentes matières grasses alimentaires et graisses de dépôt.

(**) Teneur en constituants solides (triglycérides) à différentes températures (MANSFIELD, 1971). 
En ce qui concerne les autres constituants, la composition des 5 aliments d'allaitement était absolument identique. Des expériences faites antérieurement ont montré (CoTTYN et al., I969) que l'on peut remplacer ro $\mathrm{p}$. Ioo de la poudre de lait écrémé par de la poudre de lactosérum. L'incorporation des matières grasses dans les aliments d'allaitement a été effectuée par voie sèche. Dans cette méthode, il importe d'obtenir un mélange aussi intime que possible entre la poudre de lait écrémé (phase solide) et les matières grasses (phase liquide). L'emploi d'un émulsifiant adéquat joue un rôle important en favorisant l'émulsification lors de la reconstitution du lait de remplacement. Lors d'essais antérieurs, l'addition d'un émulsifiant du type saccharoglycérides a eu un effet favorable sur la croissance (Cortys et al., I969). Nous avons donc ajouté 3,I g de MST I I $(80,20 \mathrm{~L})$ (mélange industriel contenant $60 \mathrm{p}$. Ioo de saccharoglycérides et $20 \mathrm{p}$. Ioo de lécithine) et $\mathrm{r}, 4 \mathrm{~g}$ de lécithine de soja $65 \mathrm{p}$. Ioo par Ioo $\mathrm{g}$ de mélange de matières grasses. L'adjonction de cet émulsifiant exerce également une influence favorable sur la digestibilité des matières grasses (Toullec et MATHIEU, I970).

Nous avons utilisé uniquement des matières grasses raffinées. En ce qui concerne la qualité de ces matières grasses (tabl. 3), les exigences retenues étaient les suivantes : acidité : maximum o,r p. I0o; indice de peroxyde < I; teneur en eau et impuretés : maximum o, I p. Ioo; point de fusion $<50^{\circ} \mathrm{C}$; insaponifiable : maximum 2 p. roo. D'après Toullec (I967) il est difficile dans l'état actuel des connaissances, de formuler des critères précis de qualité, car l'utilisation métabolique des différents produits ayant subi une altération normale (acidité, indice de peroxyde etc.) est très mal connue.

\section{TABLEAU 4} Composition en acides gras des matières grasses alimentaires (I)
et des graisses de dépôt (2)

\begin{tabular}{|c|c|c|c|c|c|c|c|c|c|c|}
\hline \multirow{2}{*}{$\begin{array}{c}\text { Composition } \\
\text { des matières grasses }\end{array}$} & \multicolumn{2}{|c|}{ Suif } & \multicolumn{2}{|c|}{ Saindoux } & \multicolumn{2}{|c|}{ BPCS } & \multicolumn{2}{|c|}{$\mathrm{PCB}$} & \multicolumn{2}{|c|}{ SoPSB } \\
\hline & (1) & (2) & (1) & (2) & (1) & (2) & (1) & $(2)$ & (1) & (2) \\
\hline$C_{8}: 0 \ldots$. & traces & 一 & traces & $\ldots$ & 1,99 & 0,21 & 1,36 & traces & traces & traces \\
\hline $\mathrm{C}_{10}: \mathbf{0} \ldots \ldots \ldots$ & 0,15 & traces & traces & traces & 1,09 & 0,42 & 0,85 & traces & 0,53 & traces \\
\hline $\mathrm{C}_{12}: \mathrm{o}_{0} \ldots \ldots \ldots$ & 0,62 & 0,15 & 0,75 & 0,20 & 9,40 & 1,41 & 7,56 & 1,88 & 1,83 & 0,66 \\
\hline $\begin{array}{l}\mathrm{C}_{14}:{ }_{0} \ldots \ldots \ldots \ldots \\
\mathrm{C}_{14}: 1 \\
\mathrm{C}_{14}\end{array}$ & 5,25 & 2,87 & 2,91 & 2,40 & 5,48 & 4,88 & 6,65 & 8,90 & 5,42 & 5,33 \\
\hline$+c_{15} \ldots \ldots$ & 2,51 & 1,41 & traces & 0,80 & 1,55 & 1,89 & 0,80 & 1,52 & 1,54 & 1,27 \\
\hline $\begin{array}{l}C_{16}: 0+C_{16} \text { iso } \ldots \\
C_{16}: C_{17}\end{array}$ & 20,73 & 21,89 & 22,23 & 21,98 & 23,00 & 28,15 & 21,37 & $27,0 \prime_{t}^{\prime}$ & 21,80 & 21,69 \\
\hline $\begin{array}{r}16: \\
+\mathrm{C}_{17} \text { iso } \ldots . . .\end{array}$ & $2,6 \mathbf{t}$ & 7,30 & 4,22 & 5,53 & 5,34 & 2,99 & 7,49 & 6,50 & 6,96 & 6,86 \\
\hline $\mathrm{C}_{18}: 0+\mathrm{C}_{18}$ iso $\ldots$ & 24,57 & 18,63 & 20,50 & 17,20 & 14,02 & 19,21 & 10,82 & 13,66 & 11,79 & 16,82 \\
\hline$C_{18}: x \ldots \ldots$ & 34,70 & $41,6 / \mathrm{k}$ & 39,15 & 46,15 & 29,89 & 36,27 & 27,90 & 33,15 & 29,66 & 36,60 \\
\hline $\mathrm{C}_{18: 2} \ldots \ldots$ & 4,83 & 4,32 & 7,36 & 5,07 & 4,15 & 3,20 & 5,08 & 2,79 & 7,50 & 6,66 \\
\hline$C_{18: 3}+C_{20: 1} \ldots$ & 2,91 & - & 2,85 & - & 1,93 & 1,31 & 4,80 & 3,06 & 5,89 & 1,09 \\
\hline $\mathrm{C}_{20}: 0+\mathrm{C}_{20}$ poly $\ldots$ & $1,0^{4}$ & 1,73 & traces & 0,62 & 1,56 & - & 2,88 & 0,64 & 3,09 & 2,96 \\
\hline $\mathrm{C}_{22}: 0+\mathrm{C}_{22: 1} \ldots$ & - & - & - & - & 0,51 & - & 2,36 & 0,79 & 3,93 & $\longrightarrow$ \\
\hline
\end{tabular}

Pour ce qui est de la composition en acides gras, (tabl. 4) les normes recherchées étaient les suivantes : $\mathrm{C}_{12}:$ o (acide laurique) $: 5$ à Io p. Ioo; $\mathrm{C}_{14: 0}$ (acide myristique) : 4 à $6 ; \mathrm{C}_{16}: 0$ (acide palmitique) : maximum $30 ; \mathrm{C}_{18: 0}$ (acide stéarique) : maximum $15 ; \mathrm{C}_{18: 1}$ (acide oléique) : maximum $35 ; \mathrm{C}_{18: 2}$ (acide linoléique) $+\mathrm{C}_{18: 3}$ (acide linolénique) $: 3$ à Io.

Le suif et le saindoux ne répondent pas aux normes recherchées à cause d'une trop faible teneur en acides gras moyens (acides laurique et myristique) ; lour teneur en acide stéarique est également supérieure à la norme maximum de I5. A cause de la présence de ro p. Ioo d'huile de soja dans le mélange SoPSB, la teneur en acides gras polyinsaturés dépassait Io p. Ioo. 


\section{Méthodes d'analyse}

Analyse du sang.

Valeur de l'hématocrite.

Celle-ci a été déterminée dans des tubes à hématocrite suivant la méthode de Wintrobe, en centrifugeant pendant une demi-heure à 3500 tours/minute du sang hépariné. La hauteur de la masse d'érythrocytes, en p. Ioo de la hauteur totale des cellules du plasma, donne la valeur de l'hématocrite.

\section{Hémoglobine.}

L'hémoglobine a été dosée par la méthode de l'oxyhémoglobine à l'aide d'un hémoglobinemètre Spencer qui mesure l'absorption de la lumière par l'oxyhémoglobine en employant un filtre vert (ScHALM, I967).

Analyse de la viande.

Détermination de la couleur par voie physique.

La couleur a été déterminée à l'aide d'un photomètre tristimuli (Hunterlab color and color difference meter) sur une coupe fraîche du longissimus dorsi (long dorsal) au niveau de la $6^{\mathrm{e}}$ côte. La valeur indiquée par l'appareil est une mesure de la "clarté " de l'échantillon de viande. Cette valeur est d'autant plus élevée que la viande est plus pâle.

Détermination des pigments de couleur par voie chimique.

La myoglobine et l'hémoglobine résiduelle ont été déterminées sous forme d'hématine après extraction à l'acétone et à l'acide chlorhydrique. La teneur en hématine est d'autant plus élevée que la viande est plus foncée (Hornsey, r956).

Après avoir fait une extraction aqueuse de $10 \mathrm{~g}$ de viande on obtient, après filtration, un extrait rouge clair contenant la myoglobine et l'hémoglobine. L'absorption est ensuite mesurée à $54^{\circ} \mathrm{m} \mu$ dans des cuves où l'épaisseur de la couche est de I cm; elle est exprimée en valeur d'extinction. Le coefficient d'extinction ou d'extraction est d'autant plus élevé que la viande est plus foncée.

\section{III. — RÉSULTATS}

\section{Production de viande}

Le tableau 5 donne un résumé des performances des veaux. Dans le groupe expérimental BPCS un animal est décédé après Io jours d'expérience à la suite d'une forte diarrhée et un autre devait être éliminé après 98 jours à cause d'une broncho-pneumonie.

Par rapport au suif ou au saindoux purs, les mélanges de matières grasses ont favorablement influencé la croissance journalière et l'indice de consommation. La croissance journalière la plus forte ( I I69 g) ainsi que l'indice de consommation le plus favorable $(I, 56)$ ont été obtenus avec le mélange PCB. La formule BPCS a donné une croissance journalière un peu moins élevée ( $I$ I55 g) et un indice de consommation quasi identique $(I, 57)$. Le tableau 6 donne l'évolution de la croissance journalière et de l'indice de consommation durant les périodes expérimentales successives.

La différence finale dans le rythme de croissance entre les mélanges PCB et BPCS se manifeste nettement au cours de la dernière période expérimentale ( 16 semaines-fin), à savoir I 583 contre I $508 \mathrm{~g} /$ jour. La suppression de l'huile de coprah 


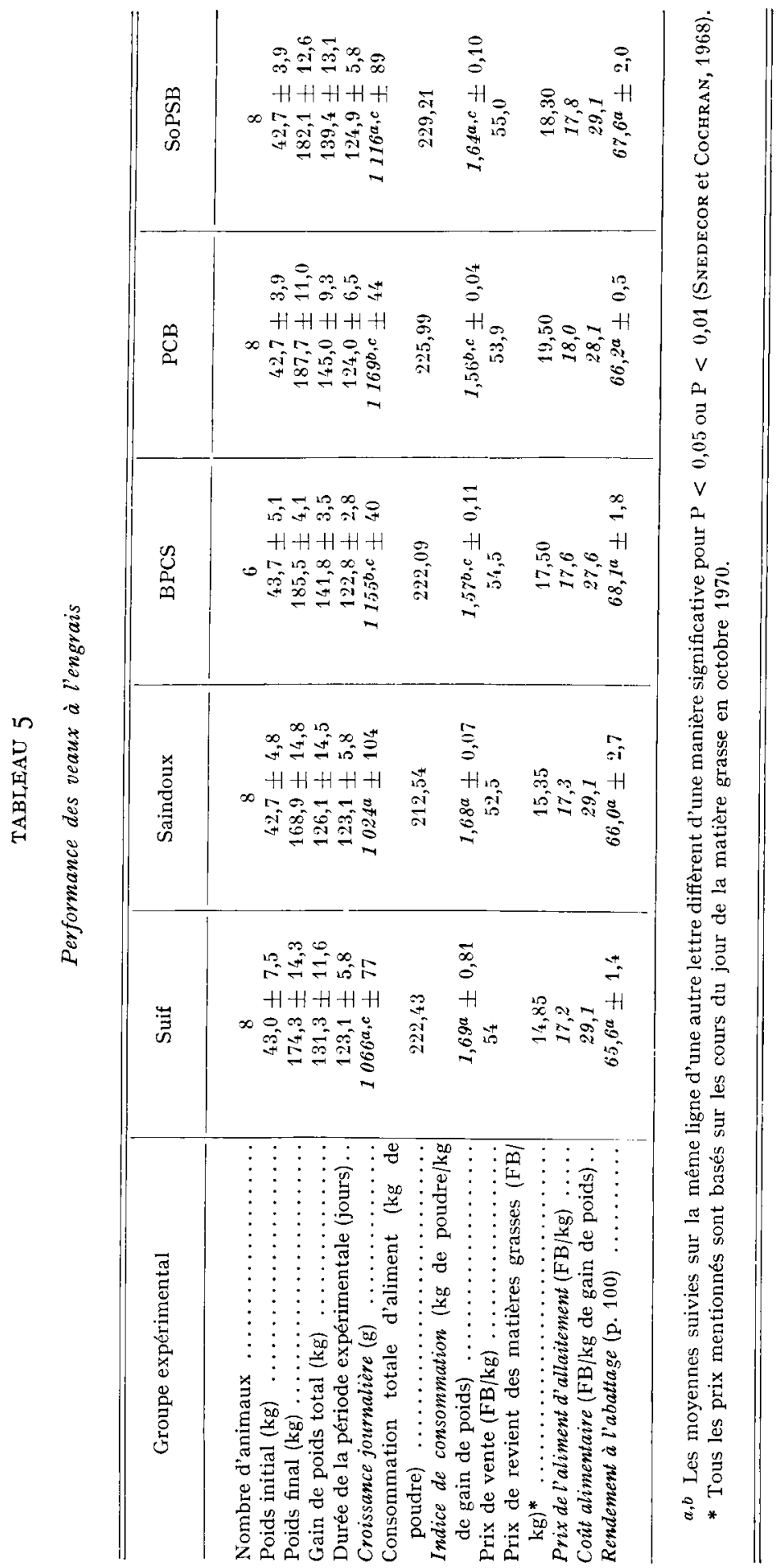


et son remplacement partiel par de 1'huile de soja (SoPSB) a quelque peu réduit la croissance journalière ( I II6 g) tout en influençant défavorablement l'indice de consommation $(I, 64)$. Ces deux influences défavorables n'étaient cependant pas significatives.

\section{TABLEAU 6}

\section{Évolution de la croissance journalière et de l'indice de consommation} durant les semaines expérimentales successives

I. - Croissance journalière $(\mathrm{g})$

\begin{tabular}{|c|c|c|c|c|c|}
\hline & Suif & Saindoux & BPCS & PCB & SoPSB \\
\hline \& premières semaines.... & $388 \pm 92$ & $370 \pm 144$ & $164 \pm 55$ & $469 \pm 70$ & $357 \pm 87$ \\
\hline ' -8 semaines $\ldots \ldots \ldots$ & $1116 \pm 85$ & $1165 \pm 129$ & $1250 \pm 84$ & $12 / 5 \pm 110$ & $1210 \pm 148$ \\
\hline 8-12 semaines $\ldots \ldots \ldots$ & $1196 \pm 87$ & $1214 \pm 253$ & $1333 \pm 80$ & $1321 \pm 101$ & $1281 \pm 92$ \\
\hline $12-16$ semaines $\ldots \ldots \ldots$ & $1^{\prime}+6 \pm 181$ & $1210 \pm 42 y_{t}^{\prime}$ & $1434 \pm 114$ & $146^{\prime} \pm 150$ & $1446 \pm 15^{\prime}$ \\
\hline 16 semaines-fin $\ldots \ldots \ldots$ & $1359 \pm 321$ & $1371 \pm 470$ & $1508 \pm 245$ & $1583 \pm 272$ & $1485 \pm 170$ \\
\hline $0-8$ semaines $\ldots$. & $752 \pm 8^{\prime}$ & $768 \pm 95$ & $857 \pm 59$ & $857 \pm 73$ & $783 \pm 102$ \\
\hline 0-12 semaines $\ldots \ldots \ldots$ & $900 \pm 5^{\prime}$ & $917 \pm 111$ & $1016 \pm 62$ & $1012 \pm 59$ & $949 \pm 91$ \\
\hline $0-16$ semaines $\ldots \ldots \ldots$ & $1037 \pm 79$ & $990 \pm 149$ & $1120 \pm 45$ & $1125 \pm 39$ & $1074 \pm 92$ \\
\hline $0-f i n \ldots \ldots \ldots \ldots \ldots$ & $1066 \pm 77$ & $1024 \pm 10^{\prime}$ & $1155 \pm 40$ & $1169 \pm 41$ & $1116 \pm 89$ \\
\hline
\end{tabular}

2. - Indice de consommation ( $\mathrm{kg}$ d'aliment/kg gain de poids)

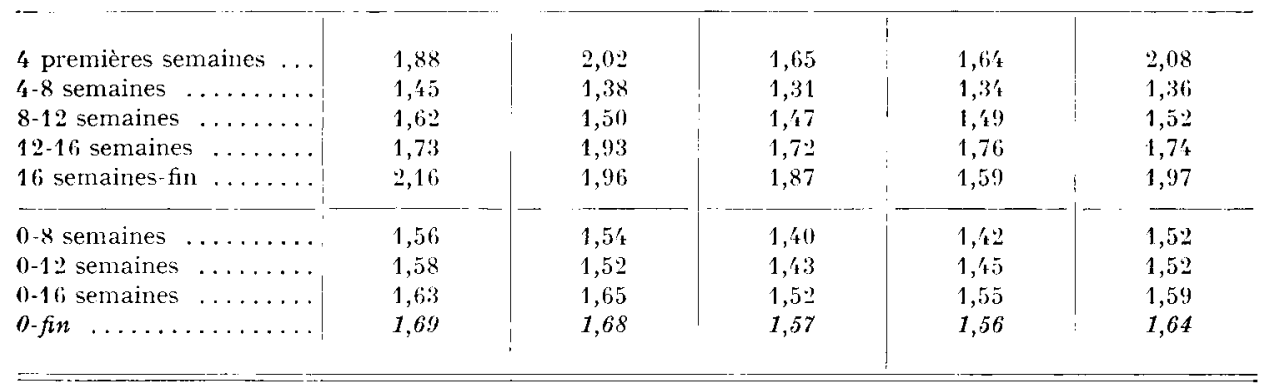

Comparé aux trois mélanges, l'emploi du suif ou du saindoux purs a donné des résultats moins satisfaisants. Avec l'aliment renfermant le suif pur, la croissance journalière a été de $\mathrm{I} 066 \mathrm{~g}$ mais c'est avec l'aliment contenant le saindoux pur que le rythme de croissance a été le plus bas ( $\mathrm{I} 024 \mathrm{~g} / \mathrm{j}$ ). Il est à noter que la différence de croissance journalière entre les deux aliments renfermant respectivement le suif et le saindoux purs, se manifeste finalement au cours de la période expérimentale I2I6 semaines (I 446 contre I 2 Io g/j) (tabl. 6). Par rapport au rythme de croissance obtenu avec les rations $\mathrm{PCB}$ et $\mathrm{BPCS}$, celui enregistré avec le saindoux était significativement moindre (respectivement pour $\mathrm{P}<0$, or et $\mathrm{P}<0,05$ ).

L'évolution de la croissance obtenue avec les 5 aliments est représentée dans la figure $\mathrm{I}$.

L'efficacité alimentaire enregistrée, tant avec l'aliment contenant le saindoux 


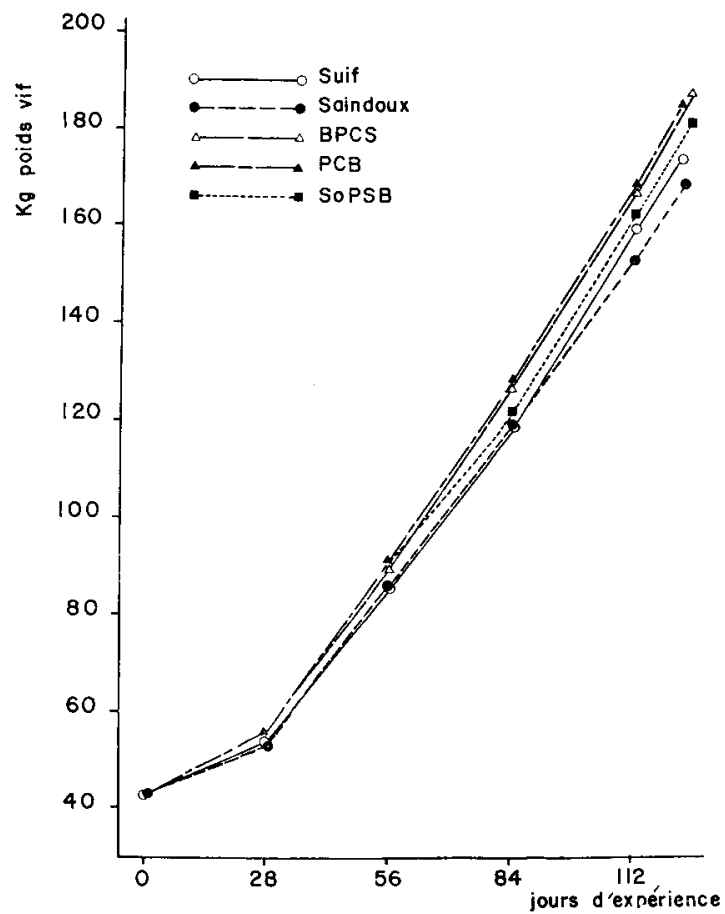

FIG. I. - Courbe de croissance des veaux à l'engrais

TABLEAU 7

Résultats concernant la formule sanguine et la couleur de la viande

\begin{tabular}{|c|c|c|c|c|c|c|c|c|c|c|c|c|c|c|c|}
\hline \multirow{2}{*}{$\begin{array}{c}\text { Groupe expérimental } \\
\\
\text { Nombre de veaux } \ldots \ldots \\
\text { Poids initial }(\mathrm{kg}) \ldots \ldots\end{array}$} & \multicolumn{3}{|c|}{$100 \%$ suif } & \multicolumn{3}{|c|}{$\begin{array}{c}100 \% \\
\text { saindoux }\end{array}$} & \multicolumn{3}{|c|}{ BPCS } & \multicolumn{3}{|c|}{ PCB } & \multicolumn{3}{|c|}{ SoPSB } \\
\hline & & 8 & & & 8 & & & 6 & & & 8 & & & 8 & \\
\hline $\begin{array}{c}\text { Poids initial }(\mathrm{kg}) \ldots \ldots \\
\text { Formule sanguine } \\
\text { Hématocrite }\end{array}$ & 43,0 & \pm & 7,5 & 42,7 & 土 & 4,8 & $1 / 3,7$ & \pm & 5,1 & 42,7 & \pm & 3,9 & 42,7 & \pm & 3,9 \\
\hline $\begin{array}{c}\text { Valeur initiale } \ldots \ldots \ldots \\
\text { - finale. .......... } \\
\text { Hémoglobine } \\
(\mathrm{g} / 100 \mathrm{ml} \text { sang })\end{array}$ & $\begin{array}{l}45,2 \\
35,0\end{array}$ & $\begin{array}{l} \pm \\
\pm\end{array}$ & $\begin{array}{l}9,7 \\
3,0\end{array}$ & $\begin{array}{l}45,0 \\
30,1\end{array}$ & $\begin{array}{l} \pm \\
\pm\end{array}$ & $\begin{array}{l}6,5 \\
\mathbf{4}, 1\end{array}$ & $\begin{array}{l}47,1 \\
30,8\end{array}$ & $\frac{ \pm}{ \pm}$ & $\begin{array}{l}7,7 \\
3,4\end{array}$ & $\begin{array}{l}45,1 \\
33,8\end{array}$ & $\frac{ \pm}{ \pm}$ & $\begin{array}{l}7,6 \\
4,7\end{array}$ & $\begin{array}{l}45,0 \\
33,2\end{array}$ & $\begin{array}{l} \pm \\
\pm\end{array}$ & $\begin{array}{l}7, \mathbf{x} \\
4,5\end{array}$ \\
\hline $\begin{array}{l}\text { Valeur-initiale } \ldots \ldots \\
\quad-\quad \text { finale } . . . \\
\text { Couleur de la viande }\end{array}$ & $\begin{array}{r}12,4 \\
9,9\end{array}$ & $\begin{array}{l} \pm \\
\pm\end{array}$ & $\begin{array}{l}2,4 \\
1,1\end{array}$ & $\begin{array}{r}12,1 \\
8,8\end{array}$ & $\begin{array}{l} \pm \\
\pm\end{array}$ & $\begin{array}{l}1,6 \\
1,4\end{array}$ & $\begin{array}{r}12,2 \\
8,9\end{array}$ & $\begin{array}{l} \pm \\
\pm\end{array}$ & $\begin{array}{l}1,6 \\
1,5\end{array}$ & $\begin{array}{r}11,8 \\
9,8\end{array}$ & $\frac{ \pm}{ \pm}$ & $\begin{array}{l}1,4 \\
1,4\end{array}$ & $\begin{array}{r}12,1 \\
9,4\end{array}$ & $\begin{array}{l} \pm \\
\pm\end{array}$ & $\begin{array}{l}0,95 \\
1,2\end{array}$ \\
\hline $\begin{array}{l}\text { Hunter (valeur-L) } \ldots \ldots \\
\text { Hématine (p.p.m.) } \ldots \ldots \\
\text { Coefficient d'extinction }\end{array}$ & $\begin{array}{r}46,7 \\
57,1 \\
0,184\end{array}$ & $\begin{array}{l} \pm \\
\pm \\
\pm\end{array}$ & $\begin{array}{l}4,3 \\
10,3 \\
0,04\end{array}$ & $\begin{array}{r}48,5 \\
55,7 \\
0,150\end{array}$ & $\begin{array}{l} \pm \\
\pm \\
\pm\end{array}$ & $\begin{array}{l}3,7 \\
11,6 \\
0,03\end{array}$ & $\begin{array}{r}49,3 \\
49,5 \\
0,146\end{array}$ & $\begin{array}{l} \pm \\
\pm \\
\pm\end{array}$ & $\begin{array}{l}5,3 \\
12,2 \\
0,07\end{array}$ & $\begin{array}{r}49,4 \\
53,7 \\
0,144\end{array}$ & $\begin{array}{l} \pm \\
\pm \\
\pm\end{array}$ & $\begin{array}{l}1,5 \\
11,6 \\
0,03\end{array}$ & $\begin{array}{r}48,9 \\
51,6 \\
0,148\end{array}$ & $\begin{array}{l} \pm \\
\pm \\
\pm\end{array}$ & $\begin{array}{l}4,6 \\
10,8 \\
0,05\end{array}$ \\
\hline
\end{tabular}

Les moyennes figurant sur la même ligne ne présentent pas de différence significative pour $P<0,05$ 
qu'avec celui renfermant le suif, était significativement moins bonne $(\mathrm{P}<0,5)$ que celle obtenue avec les aliments PCB et BPCS. Compte tenu du prix de revient des différentes matières grasses ou mélanges de matières grasses, ainsi que de celui des différents éléments nutritifs incorporés, 1e prix de revient de base des différents aliments d'allaitement variait de I7,2 à I 8 F. B. $/ \mathrm{kg}$. Grâce à 1 'indice de consommation sensiblement meilleur avec les aliments PCB et BPCS, les coûts alimentaires par $\mathrm{kg}$ de gain de poids étaient sensiblement moindres pour ces aliments (resp. 27,6 F. B. et $28, \mathrm{I} \mathrm{F} . \mathrm{B} . / \mathrm{kg}$ ) que pour les trois autres, où ils atteignaient uniformément $29, \mathrm{I}$ F. B. Le rendement à l'abattage, satisfaisant dans l'ensemble, ne révélait pas de différences significatives entre les 5 aliments.

\section{Qualité de la viande}

Après I7 semaines d'expérience en moyenne, les veaux étaient abattus dans un abattoir situé non loin de la station ; au préalable, une prise de sang était effectuée le matin en vue de la détermination de la valeur de 1'hématocrite et de la teneur en hémoglobine. Environ $24 \mathrm{~h}$ après l'abattage, un morceau du longissimus dorsi (long dorsal) était prélevé au niveau de la $6^{\text {e }}$ côte pour être analysé au laboratoire. En ce qui concerne la qualité de la viande, il n'y a pas eu de différences significatives entre les différents groupes (tab1. 7).

\section{Infuence des matières grasses alimentaires sur les graisses des dépôts adipeux.}

Le tableau 3 donne le rapport existant entre la composition des matières grasses alimentaires et celle des graisses des dépôts. Par rapport aux matières grasses alimentaires, les graisses des dépôts ont une teneur relativement élevée en acides gras libres. Il convient de remarquer que la fonte de la graisse de dépôt ( 24 heures à $100^{\circ} \mathrm{C}$ ) et sa filtration allaient de pair avec une augmentation du degré d'acidité et de l'indice de peroxyde. Des traces d'eau, de sang, d'albumine, etc., catalysent l'augmentation de la teneur en acides gras. La valeur de l'indice de peroxyde est élevée même si l'on tient compte du fait que la fonte influence défavorablement cet indice. On peut donc admettre que des graisses raffinées ont un indice de peroxyde moindre que des graisses fondues par voie naturelle. Ceci se remarque également lors du test de stabilité selon SwIFT : la graisse raffinée présente une meilleure résistance à 1'oxydation que la graisse de dépôt brute. On doit remarquer que les matières grasses alimentaires avaient également été stabilisées par addition de $B H^{\prime} T$, ce qui n'était pas le cas des graisses de dépôt. L'indice d'iode des matières grasses alimentaires est inversement proportionnel à celui des matières grasses des dépôts (fig. 2). En effet, exception faite pour le saindoux, à une graisse alimentaire à indice d'iode bas correspond une graisse de dépôt à indice d'iode plus élevé et réciproquement.

La valeur de l'indice de saponification est chaque fois plus élevée dans les graisses alimentaires que dans les graisses des dépôts correspondantes. Il semblerait que les acides gras alimentaires les plus courts soient dissociés et utilisés pour la synthèse de chaînes plus longues qu'on retrouve dans les dépôts.

Le pourcentage de triglycérides solides (résonance magnétique nucléaire), que l'on retrouve à des températures déterminées dans les graisses de dépôt des veaux dont les aliments renfermaient le suif ou le saindoux, est chaque fois sensiblement moins élevé que celui des graisses alimentaires correspondantes (tabl. 3, fig. 3). 
Avec les 3 mélanges de matières grasses, les pourcentages de triglycérides solides se rapprochent toutefois de ceux des matières grasses alimentaires correspondantes (tab1. 3, fig. 3).

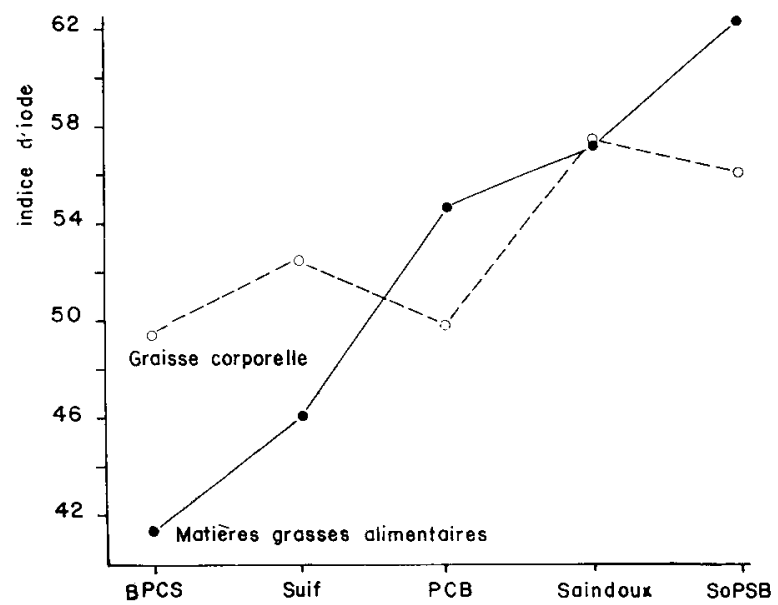

FIG. 2. - Indice d'iode des matières grasses alimentaires et des graisses corporelles

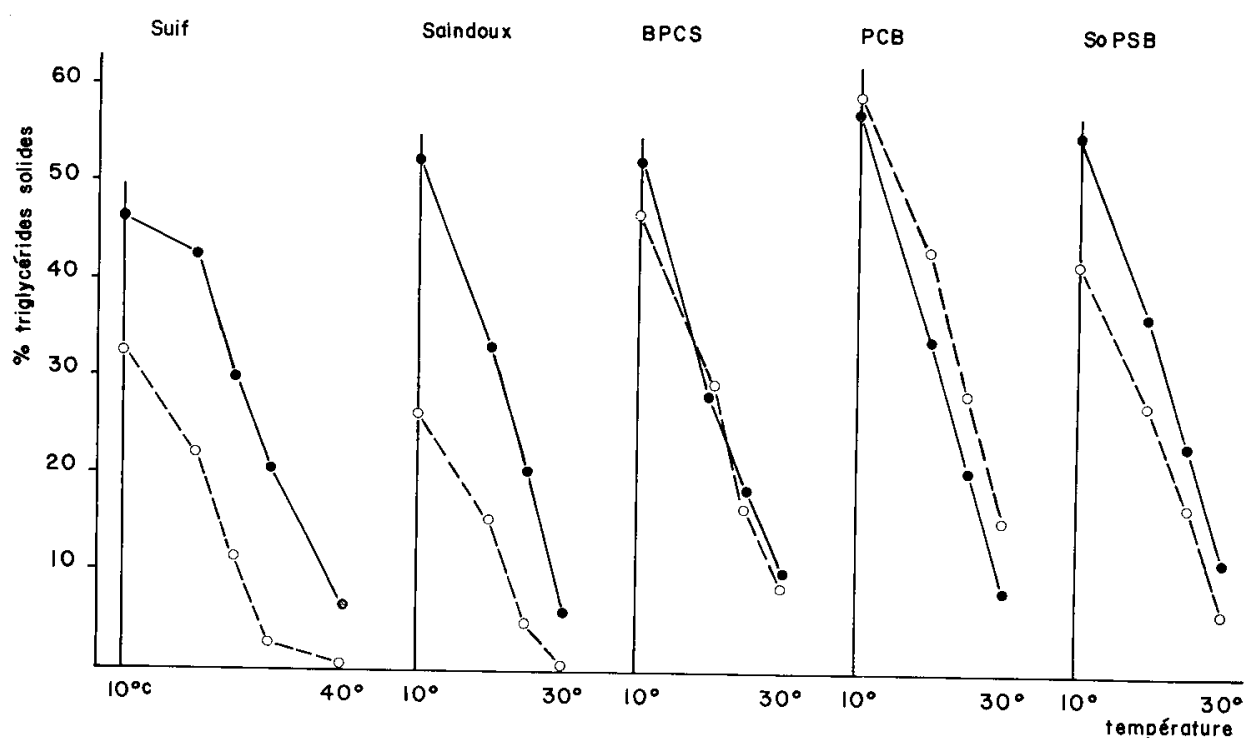

Fig. 3. - Résonance magnétique nucléaire

Aussi la somme totale des triglycérides solides, trouvée aux différentes températures enregistrées, était-elle sensiblement plus élevée dans ces dernières graisses des dépôts que dans les premières. Des veaux ayant reçu le suif ou le saindoux ont donc des graisses de dépôt moins fermes que celles des animaux ayant consommé les mélanges de matières grasses. 
Le tableau 4 donne le rapport entre la composition en acides gras des matières grasses alimentaires et celle des graisses des dépôts correspondantes. Les acides gras à chaîne courte $\left(\mathrm{C}_{8}\right.$ à $\mathrm{C}_{12}$ inclus $)$ des matières grasses alimentaires ne sont pratiquement plus retrouvés dans les graisses de dépôts. Les chaînes longues $\left(\mathrm{C}_{14}\right.$ à $\left.\mathrm{C}_{18}\right)$ inclus sont largement déposées. D'autre part il semble que les acides gras saturés, ceux à chaîne courte exceptés, soient retrouvés en régle générale dans les graisses de dépôt. Le pourcentage d'acides gras mono-insaturés (surtout d'acide oléique) est plus élevé dans les graisses des dépôts que dans les matières grasses alimentaires correspondantes. Parmi les acides gras polyinsaturés, seul l'acide linoléique est stocké en partie dans les graisses de réserve. Nous n'avons pas pu établir s'il s'agit ici d'une forme cis-cis de l'acide linoléique.

\section{IV. - DISCUSSION ET CONCLUSION}

L'huile de baleine partiellement hydrogénée peut constituer jusqu'à 60 p. Ioo des matières grasses totales incorporées dans les aliments d'allaitement pour veaux à l'engrais (tabl. 5). Du point de vue production de viande, les résultats obtenus avec le mélange $\mathrm{PCB}$, renfermant 60 p. Ioo d'huile de baleine, étaient même un peu plus favorables que ceux obtenus avec la formule BPCS, dans laquelle avaient été incorporés 50 p. Ioo de suif et Io p. Ioo d'huile de baleine. BAKKER et VEEN (I969) ont montré que, tant en ce qui concerne la digestibilité que l'effet sur la croissance, l'huile de baleine hydrogénée de manière sélective vaut le saindoux ou un mélange de saindoux, de suif et d'huile de palme. Du fait que le veau se comporte sur le plan fonctionnel comme un monogastrique, il n'y a pas chez lui comme chez les ruminants une hydrogénation des acides gras insaturés; en conséquence, lorsque l'aliment contient des acides gras polyinsaturés, son utilisation métabolique semble moins bonne, il s'ensuit une faible digestibilité de ces matières grasses alimentaires et des cas fréquents de diarrhée. Il a été montré que 1'hydrogénation partielle améliore sensiblement la digestibilité et l'utilisation de ces matières grasses par le veau (BAKKER et VEEN, 1969). Des huiles marines ou de poisson non hydrogénées, telle que celle de baleine, ne se prêtent normalement pas à une incorporation dans les mélanges de matières grasses pour veaux à l'engrais, étant donné leur faible digestibilité et le danger de diarrhée qu'elles entraînent. Une hydrogénation complète rend le point de fusion trop élevé, de sorte que la digestibilité diminue. Dans notre essai, l'hydrogénation avait été réalisée jusqu'à un point de fusion de $3^{\circ} \mathrm{C}$.

Du point de vue physiologie nutritionnelle, l'huile de coprah et de palmiste est très bien utilisée à cause de sa forte teneur en acides gras à chaîne courte et sa faible teneur en acides gras insaturés ; à ce propos, elle présente une certaine similitude avec les matières grasses du lait. D'après Toul_EC et MATHIEU (I969), il semble que ce sont surtout les matières grasses à forte teneur en acides gras à chaînes courtes qui, en tant que source d'énergie très digestible, conviennent au veau à l'engrais durant les premières semaines de sa vie.

A ce sujet, VEEN (I970) fait remarquer que des acides gras avec I2 ou moins d'atomes de carbone gagnent surtout le foie par la veine porte, tandis que les acides gras à longue chaîne gagnent la circulation sanguine par les vaisseaux 1ymphatiques. 
De cette manière, un pourcentage élevé des matières grasses du " type laurique » devient immédiatement et facilement disponible comme source d'énergie.

L'huile de coprah et de palmiste a surtout une forte teneur en acide laurique (45-5 I p. Ioo) et en acide myristique (I6,5-I8,5 p. IOo), tandis que l'huile de palme, qui a une forte teneur en acide oléique $C_{18: 1}(43-50 \mathrm{p}$. IOo) et en acide linoléique $\mathrm{C}_{18: 2}$ (5-Io p. IOO), contient également beaucoup d'acide palmitique $\mathrm{C}_{16: 0}$ (36-43 p. I00). L'institut de Schothorsт (I966) a réalisé des essais comparant un mélange de suif et de saindoux au même mélange additionné de proportions croissantes d'huile de palmiste ( $12,5,25$ et 50 p. roo). La croissance et l'indice de consommation étaient régulièrement améliorés par les doses croissantes d'huile de palmiste ; par conséquent, dans certains cas, la présence d'acides gras à chaîne courte exerce un effet favorable. L'incorporation, dans la dernière formule, de Io p. Ioo d'huile de soja en remplacement partiel de l'huile de coprah a influencé défavorablement la production de viande. La forte teneur de l'huile de soja en acides gras polyinsaturés, (acide linoléique : 49-55 p. Ioo et acide linoléique 4-I2 p. IOO), avait pour effet de porter la somme de ces derniers à I3,4 (tab1. 4), dépassant de la sorte la norme maximum de ro que nous avons citée plus haut. La norme minimum acide linoléique + acide linolénique $>3$ était d'ailleurs largement remplie dans les autres formules. Les résultats défavorables obtenus avec le saindoux semblent quelque peu étonnants, surtout en ce qui concerne la comparaison avec le suif.

D'après TOULLEC et MATHIEU (I969) le saindoux est sensiblement plus digestible que le suif ( 96 au lieu de 90 p. Ioo). Selon Dimick et al. (I965), les matières grasses du lait et le saindoux ont en outre une structure triglycéridique caractéristique et comparable, dans laquelle l'acide plamitique présente de préférence une disposition interne dans les triglycérides de poids moléculaire élevé. Avec des aliments contenant respectivement 18,8 p. Ioo de suif ou de saindoux pur, RoGNoni et Aмrch Gal, ( 1967 ) ont cependant obtenu dans l'intervalle de poids $5^{8-158} \mathrm{~kg}$ des gains de poids journaliers ( $\mathrm{I}$ o8o et $\mathrm{I} 087 \mathrm{~g}$ ) et des indices de consommation $(\mathrm{I}, 77$ et $\mathrm{I}, 74)$ pratiquement identiques.

La composition en acides gras des graisses de dépôt est fortement influencée par celle des matières grasses alimentaires. Ceci est en concordance avec les observations rapportées par Toul_LEC et Mathieu (I969). De même que chez le monogastrique, la composition des graisses de dépôt du veau à l'engrais dépend de celle des matières grasses alimentaires. L,e veau à l'engrais diffère à ce sujet du ruminant chez lequel, à cause du processus d'hydrogénation dans le rumen, les graisses corporelles ont une composition relativement constante. Le veau à l'engrais est capable de métaboliser rapidement les acides gras à chaîne courte, dont on ne retrouve dès lors que des quantités minimes dans les graisses de réserve. Aussi les mélanges de matières grasses qui, par analogie avec les matières grasses du lait, renferment un pourcentage important d'acides gras courts et moyens, sont-ils très rapidement utilisés à des fins énergétiques (SCHEig et KLATSKIN, I968; AuroussEau et al. I97o; Vermorel et Aurousseau, I970 ; Aurousseau et al. I970). Les acides gras saturés à longue chaîne sont moins rapidement métabolisés et sont davantage stockés dans les graisses de réserve. Les acides gras à longue chaîne devraient favoriser l'engraissement au cours de la deuxème période de la vie ('Touı_fic et MATHIEU, I969). Les acides gras polyinsaturés ont été généralement peu stockés dans les graisses de dépôt.

Par rapport à 1'incorporation à 1'aliment d'allaitement d'un mélange de matières 
grasses végétales et animales, celle de matières grasses exclusivement animales semble donner une viande d'une couleur un peu moins claire. Bien que l'analyse de la viande n'ait porté que sur 6 veaux et que de ce fait les différences observées ne soient pas statistiquement sûres, il semble néanmoins que 1'engraissement des veaux avec des aliments d'allaitement contenant des mélanges de matières grasses végétales et animales, donne une viande dont la couleur est très satisfaisante (BPCS). En résumé, l'utilisation de divers mélanges de matières grasses dans les aliments d'allaitement pour veaux à l'engrais n'a pas entraîné de différences significatives, bien que l'incorporation de matières grasses végétales ait eu tendance à améliorer la couleur de la viande (couleur plus claire).

Nous pouvons en conclure que le suif et le saindoux, matières grasses qui, pour des raisons économiques, sont les plus employées dans la préparation des aliments d'allaitement pour veaux à l'engrais, peuvent avantageusement être remplacées par des mélanges de matières grasses végétales ou animales. Dans le présent essai, de fortes proportions d'huile de baleine hydrogénée (jusqu'à $60 \mathrm{p}$. Ioo), ainsi que des proportions moindres d'huile de palme et d'huile de coprah, ont donné des résultats favorables. Il serait intéressant d'étudier d'autres rapports et d'autres mélanges de matières grasses.

Rę̧u pour publication en décembre 1971.

\section{REMERCIEMENTS}

Nous tenons à remercier tout particulièrement MM. R. Toullec et J. FLANZY de l'Institut national de la Recherche agronomique, attachés respectivement aux Centres de Rennes et de Jouy-en-Josas pour leurs suggestions et critiques qui ont été pour nous une aide précieuse dans la réalisation de ce travail et la rédaction du manuscrit.

\section{SUMMARY}

\section{VEGETABLE AND ANIMAI, FATS IN MILK REPLACERS FOR VEAL CALVES}

Using 5 groups of 8 veal calves, we studied the influence of different fats and fat mixtures in milk replacers on performances, meat quality and body fat composition of the animals.

The first two formulas contained only tallow or lard as fat compound. The fat mixtures of the 3 other rations were composed as follows : BPCS : Io p. Ioo hydrogenated whale oil; i 5 p. Ioo palm oil, 25 p. roo coconut oil and $5^{\circ}$ p. roo tallow, PCB : I5 p. roo palm oil, 25 p. Ioo coconut oil ; 60 p. roo hydrogenated whale oil. SoPSB : ro p. roo soybean oil, 15 p. roo palm oil, I 5 p. Ioo tallow and $60 \mathrm{p}$. Ioo hydrogenated whale oil. The quantity of incorporated fat amounted always to 20 p. Ioo of the total formulation ; as regards the other components, the 5 formulas were completely identical. They were composed of $63 \mathrm{p}$. 100 spray dried skim-milk powder, $7 \mathrm{p}$. Ioo whey powder, 5 p. Ioo amygel (starch), 3 p. roo glucose (dextrose), I p. Ioo of a vitamin and mineral mixture (table I). For the preparation of the milk replacer we used an emulgator of the type " saccharo-glycerides". The emulgator was added in a concentration of 3, T g MST i r (80,20 L) (industrial mixture of ro p. roo saccharo-glycerides and $20 \mathrm{p}$. too lecithin) and $\mathrm{I}, 4 \mathrm{~g}$ soya lecithin, $65 \mathrm{p}$. Ioo to Ioo $\mathrm{g}$ fat mixture. In all preparations refined fats were always used. Quality characteristics of fats and fat mixture used are reported in table 3 .

Table 4 shows fatty acid patterns of fats and fat mixtures. Calves were slaughtered after an average experimental period of 124 days (average weight interval $43-\mathrm{I} 80 \mathrm{~kg}$ ) (table 5). 
The highest daily growth rate (I $\mathrm{I} 69 \mathrm{~g} / \mathrm{day}$ ) and the most favourable feed conversion efficiency $(1.56)$ were observed with ration PCB (I 5 p. Ioo palm oil, 25 p. Ioo coconut oil and 60 p. Ioo hydrogenated whale oil.) With ration BPCS (ro p. Ioo hydrogenated whale oil, I 5 p. Ioo palm oil, $25 \mathrm{p}$. 100 coconut oil and $50 \mathrm{p}$. Ioo tallow), a slightly lower daily gain (I I $55 \mathrm{~g} /$ day) together with an almost identical feed conversion (I.56) were observed. When removing coconut oil from the formulas and replacing it partially by soybean oil (formula SoPSB) the daily gain was somewhat depressed ( 1 I $6 \mathrm{~g} / \mathrm{day}$ ) and the feed conversion ratio (1.64) also affected unfavourably.

The lowest daily gain ( $\mathrm{I} 024 \mathrm{~g} / \mathrm{day}$ ) was noticed with the ration containing pure lard compared with the ration containing pure tallow (I $066 \mathrm{~g} / \mathrm{day}$ ). The growth rate obtained with lard was moreover significantly lower in comparison to the growth rate obtained with rations $\mathrm{PCB}$ and BPCS. The feed conversion efficiency obtained as well with lard as with tallow were significantly higher with respect to the rations $\mathrm{PCB}$ and BPCS. Considering feed cost price of the different fats or fat mixtures as well as the other incorporated nutrients, basis cost price of the different milk replacers varied between $\mathrm{I} 7.2$ and $\mathrm{I} 8 \mathrm{~F} / \mathrm{kg}$. The more favourable feed conversion obtained with the two pre-cited formulas resulted in a lower feed cost price per $\mathrm{kg}$ gain 27.6 and 28 . I F/kg respectively in comparison with the 3 other rations $(29.1 \mathrm{~F} / \mathrm{kg}$ gain). Dressing percentage amounted to $66.7 \mathrm{p}$. IOO and was not significantly different for the 5 experimental groups.

Concerning meat quality characteristics, no significant difference for the various criteria studied could be observed between the different groups (table 7). Calves fed with tallow or lard showed rather tender body fats in comparison with animals fed with the 3 combined fat mixtures, where body fats appeared to be much harder (table 3). Only small amounts of middle chain fatty acids $\left(\mathrm{C}_{8}\right.$ to $\mathrm{C}_{12}$ included) as well as polyunsaturated fatty acids are deposited in body fats. However, long chain saturated fatty acids are generally recovered in the body fat (table 4 ).

\section{RÉFÉRENCES BIBLIOGRAPHIQUES}

Aurousseau B., De Groot L., Vermorel M., I97o Étude comparée de l'utilisation énergétique de régimes riches en acide caprylique ou en acides gras insaturés. Ann. Biol. anim. Bioch. Biohys., 10, $703-706$.

Aurousseau B., De Groot L., Bouvier J.-C., Vermorel L., I97o. Utilisation métabolique des acides gras courts, moyens et longs par le rat en croissance. Energy Metabolism of Farm Animals E.A.A.P. Proc. 5th Symp. Vitznau, Switzerland, p. I90-I92.

BAKKer I. J. Tj., VeEN W. A. G., I969. L'usage de l'huile de baleine. sélectivement hydrogénée comme constituant d'un lait d'allaitement pour veaux à l'engrais. (D) bruikbaarheid van selectief geharde visolie als bestanddeel van een kunstmelkpreparaat voor mestkalveren. Rapport annuel, Institut pour l'Alimentation animale "De Schothorst " Hoogland Nederland p. 63-68.

Charpentier J., I966. Pigmentation musculaire du veau de boucherie. II. Influenced'unesupplémentation alimentaire en fer sur la teneur en fer héminique de qualques muscles. Ann. Zootech., 15, 36r-366.

Cottyn B. G., Casteels M. R., Buysse F. X., I969. Le remplacement de la poudre de lait écrémé par de la poudre de sérum de lait et de la levure S.A.V. dans les rations pour veaux de boucherie. Revue de l'A gric., Bruxelles, 22, 83-Ioo.

DE VRIES S. I., I955. Haematologisch Onderzoek p. 85. Wetenschappelijke Uitgeverij N. V. AmsterdamNederland.

Dimick P. S., Mccarthy R. D., Patton S., I965. Structure and synthesis of milk fat. VIII. Unique positioning of palmitic acid in milk fat triglycerides. J. Dairy Sci. 48, 735-736.

Eeckhout W., Casteels M., I969. L'influence de la formule sanguine et la teneur en fer d'aliment d'allaitement sur la couleur de la viande des veaux à l'engrais. (Het aanvankelijk bloedbeeld, naast het ijzergehalte van de kunstmelk, als invloedsfactoren op de vleeskleur van mestkalveren.) Communication R.V.V. (Station de Recherches pour l'Alimentation du Bétail, Gontrode-Belgique) no I8I.

HoRnsey H., I956. The color of cooked cured pork. J. Sci. Food Agric., 7, 534-540.

MAnsfiei. P. B., I97r. A new wide-line NMR analyzer and its use in determining the solid-liquid ratio in fat samples. J. Amer. Oil Chem. Soc., 48, 4-6.

Rognoni G., Amich Gali J., r967. Research on some new aspects of the use of fats in milk replacers. Scientific. Information, National Renders Association, publication $\mathrm{n}^{\circ}$ i 7 .

Schalm O. W., rg67. Veterinary Hematology (second édition) p. 94. Edited by Lea and Febiger, Philadelphia.

Scheig R., KLAtSkin G., rg68. Hepatic metabolism of $\mathbf{I}^{-14} \mathrm{C}$ octanoic and $\mathrm{I}^{-14} \mathrm{C}$ palmitic acids. $J$. Am. Oil. Ch. Soc., 45, 31-33.

SCHOTHORST DE., I966. Essai concernant l'utilité d'un haut pourcentage de l'huile de palmiste dans un aliment d'allaitement-essai KMB5. (Proef betreffende de wenselijkheid van de verwerking van een hoog 
percentage palmpitvet in het vet van het kunstmelkpreparaat : proef KMB5.) Rapportannuel, Institut pour l'Alimentation animale " De Schothorst " Hoogland-Nederland p. 59-62.

Snedecor G. W., Cochran W. G., I968. Statistical Methods, 6th ed. The Iowa State University Press, Ames Iowa.

Toullec R., 1967. Les corps gras dans les produits d'allaitement : Performance et contrôle de qualité. Journées internationales d'Information sur Les Aliments d'Allaitement pour veaux, Paris, 5 et 6 juin I 967 , p. 60-74.

Toullec R., Mathieu C.-M., I969. Utilisation digestive des matières grasses et de leurs principaux acides gras par le veau préruminant à l'engrais. Influence sur la composition corporelle. Ann. Biol. anim. Bioch. Biophys., 9, г 39-г60.

Toullec R., Mathieu C.-M., I970. Utilisation digestive par le veau préruminant des matières grasses incorporées dans les aliments d'allaitement par voie sèche avec ou sans émulsifiant. Ann. Zootech., 19, $89-92$.

VEeN W. A. G., I97o. Chemical research on milk replacers composition. International Meeting of Animal Nutrition Experts. (Ritena) p. I43-I54, Mallorca, I à 3 mai 1970.

Verbeke R., Martin J., Ig67. Contribution à l'étude de la couleur de la viande des veaux à l'engrais (Een bijdrage tot de studie van de vleeskleur bij mestkalveren.) Communication $n^{\circ} 8 S . K$. R. Faculté d'Agriculture, Université de Gand. Belgique.

Vermorel M., Aurousseau B., I970. Utilisation énergétique des acides acétique et caprylique par le rat en croissance. Energy Metabolism of Farm Animals, p. 185-189. E.A.A.P. Proc.5th Symp. Vitznau, Switzerland. 